\title{
Caspase-11-mediated endothelial pyroptosis underlies endotoxemia-induced lung injury
}

\author{
Kwong Tai Cheng, ${ }^{1}$ Shiqin Xiong, ${ }^{1}$ Zhiming Ye, ${ }^{1,2}$ Zhigang Hong, ${ }^{1}$ Anke Di, ${ }^{1}$ Kit Man Tsang, ${ }^{1}$ Xiaopei Gao, ${ }^{1}$ Shejuan An, \\ Manish Mittal, ${ }^{1}$ Stephen M. Vogel, ${ }^{1}$ Edward A. Miao, ${ }^{3}$ Jalees Rehman, ${ }^{1,4}$ and Asrar B. Malik ${ }^{1}$ \\ 'Department of Pharmacology, The University of Illinois College of Medicine, Chicago, Illinois, USA. Division of Nephrology, Guangdong General Hospital, Guangzhou, China. ${ }^{3}$ Department of Microbiology \\ and Immunology, Lineberger Comprehensive Cancer Center, and Center for Castrointestinal Biology and Disease, University of North Carolina at Chapel Hill, Chapel Hill, North Carolina, USA. ${ }^{4}$ Division of \\ Cardiology, Department of Medicine, The University of Illinois College of Medicine, Chicago, Illinois, USA
}

\begin{abstract}
Acute lung injury is a leading cause of death in bacterial sepsis due to the wholesale destruction of the lung endothelial barrier, which results in protein-rich lung edema, influx of proinflammatory leukocytes, and intractable hypoxemia. Pyroptosis is a form of programmed lytic cell death that is triggered by inflammatory caspases, but little is known about its role in EC death and acute lung injury. Here, we show that systemic exposure to the bacterial endotoxin lipopolysaccharide (LPS) causes severe endothelial pyroptosis that is mediated by the inflammatory caspases, human caspases $4 / 5$ in human ECs, or the murine homolog caspase-11 in mice in vivo. In caspase-11-deficient mice, BM transplantation with WT hematopoietic cells did not abrogate endotoxemia-induced acute lung injury, indicating a central role for nonhematopoietic caspase-11 in endotoxemia. Additionally, conditional deletion of caspase-11 in ECs reduced endotoxemia-induced lung edema, neutrophil accumulation, and death. These results establish the requisite role of endothelial pyroptosis in endotoxemic tissue injury and suggest that endothelial inflammatory caspases are an important therapeutic target for acute lung injury.
\end{abstract}

\section{Introduction}

Acute respiratory distress syndrome (ARDS), the most severe form of acute lung injury (ALI), is a devastating clinical complication of bacterial sepsis, with a mortality rate of greater than $40 \%$ (1). ALI is characterized by an exaggerated host-defense immune response in which influx of inflammatory cells, such as neutrophils and macrophages, into the lung tissue perpetuates a vicious cycle of inflammation that amplifies the accumulation of these cells (2). A central pathogenic feature underlying ALI is the breakdown of lung endothelial barrier function due to widespread EC death and disassembly of endothelial adherens junctions (2-4).

The endothelium lines the entire vascular system, and the lung endothelium in particular comprises about $50 \%$ of all lung cells and receives the entire cardiac output. Lung ECs are thus invariably exposed to circulating pathogens and bacterial endotoxins such as lipopolysaccharide (LPS), which are key for the pathogenesis of ALI (5). An intact endothelial barrier tightly controls lung vascular permeability (3) and leukocyte recruitment (6), whereas a disrupted endothelial barrier results in tissue edema, neutrophil influx, activation of procoagulant pathways, and release of proinflammatory cytokines such as IL-1 $\beta$ (2). Moderate increase in endothelial permeability during inflammation is an adaptive feature of the host defense response that helps resolve infection by enabling leukocyte transmigration, thus avoiding ALI, whereas severe infection can elicit an exaggerated inflammatory response

Authorship note: K. Cheng and S. Xiong contributed equally to this work. Conflict of interest: The authors have declared that no conflict of interest exists. Submitted: April 14, 2017; Accepted: August 24, 2017.

Reference information: J Clin Invest. 2017;127(11):4124-4135.

https://doi.org/10.1172/JCI94495. and endothelial barrier breakdown that is ultimately responsible for the high mortality of septic ALI.

Endotoxin (LPS) liberated in large amounts in the circulation after bacterial lysis initiates the septic cascade in Gramnegative bacterial infections (7). The canonical mammalian host-cell detection mechanism of LPS sensing occurs via the cellsurface TLR4 $(8,9)$. However, recent studies in macrophages have shown an additional intracellular LPS-sensing pathway that may be important for the pathogenesis of septic shock $(10,11)$. These studies showed that LPS breaching of the plasma membrane and binding of inflammatory caspases 4 and 5 in humans or the ortholog caspase-11 in mice activated macrophage pyroptosis, a form of programmed cell death resulting in rapid cell lysis (10-13). Caspase-11 cleavage of gasdermin D (Gsdmd) induces release of the active membrane pore-forming Gsdmd peptide, which leads to lytic death of cells by swelling (13-21). Studies of cytoplasmic LPS signaling have used LPS encapsulated by transfection reagents such as lipofectamine to mimic plasma membrane breaching by LPS in the setting of endotoxemia $(10,12,22)$. Further, delivery of LPS packaged within bacterial microvesicles, which fuse with the plasma membrane without causing breach, resulted in intracellular LPS release and also activated inflammatory caspase signaling and pyroptosis $(23,24)$.

Pyroptosis is a programmed cell death mechanism that is distinct from apoptosis and necrosis; each type of programmed cell is regulated by distinct signaling mechanisms (25). Apoptosis, a nonlytic form of cell death, is initiated by caspases $2,8,9$, and 10 and requires the effector caspases 3, 6, and 7 (26). Necroptosislike pyroptosis is a lytic cell death mechanism mediated by kinases such as RIPK3 (27). Although the molecular mechanisms of these types of programmed cell death differ, it is likely that 
A

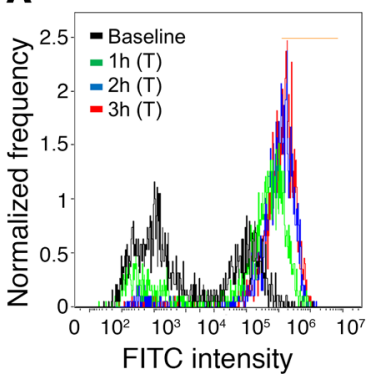

C

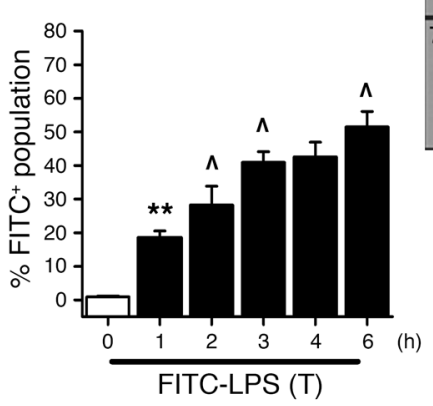

D
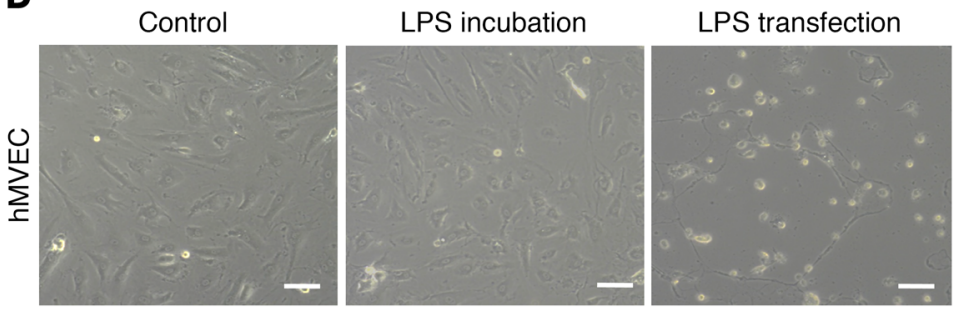

E

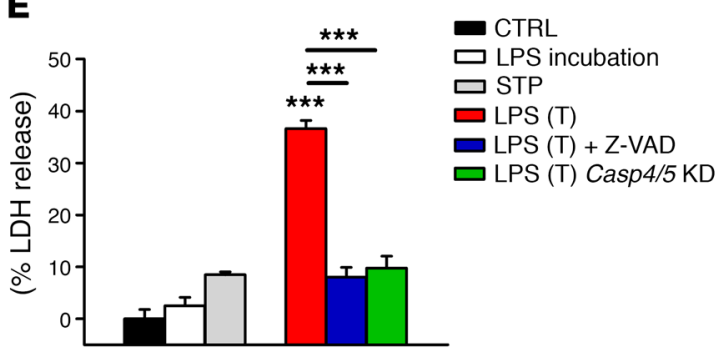

Figure 1. Intracellular LPS induces EC pyroptosis via activation of inflammatory caspases in mice and humans. (A) Flow cytometry histograms and (B) representative cytometry images of hMVECs transfected with $2 \mu \mathrm{g} / \mathrm{ml}$ FITC-labeled LPS (0111:B4) for 3 hours. LPS fluorescence in green and nuclear staining in red show that LPS crossed the EC plasma membrane. Scale bar: $20 \mu \mathrm{m}$. BF, bright field. (C) Time course of hMVEC intracellular FITC-LPS fluorescence in the presence of a transfection ( $T$ ) reagent. Results are shown as mean \pm SEM. $n=5$. ${ }^{* *} P<0.01$ from baseline; ${ }^{\wedge} P<0.01$ from previous group using ANOVA. (D) Phase-contrast micrographs of control hMVECs after a 16-hour period of LPS $(2 \mu \mathrm{g} / \mathrm{ml})$ incubation and after a 16-hour period of LPS transfection $(2 \mu \mathrm{g} /$ $\mathrm{ml}$ ) show that LPS transfection, but not LPS incubation, induced lytic cell death. Scale bars: $100 \mu \mathrm{m}$. (E) Release of LDH showed that LPS incubation $(2 \mu \mathrm{g} / \mathrm{ml})$ for 16 hours or staurosporine-induced (STP-induced) apoptosis resulted in minimal LDH release, whereas LPS transfection $(2 \mu \mathrm{g} / \mathrm{ml})$ led to marked cell lysis. Cells were primed with an initial exposure to LPS ( $500 \mathrm{ng} / \mathrm{ml}$ for 3 hours). Cell lysis was blocked by the pancaspase inhibitor Z-VAD-FMK or knockdown (KD) of the human inflammatory caspases 4 and 5 (Casp4/5). Results are shown as mean \pm SEM. $n=5 .{ }^{* *} P<0.001$ using ANOVA. the complex process of inflammation involves multiple programmed cell death pathways, depending on the type and magnitude of the inciting stimulus and cell type. From an evolutionary perspective, pyroptosis of cells harboring intracellular bacteria or in which LPS has breached the plasma membrane is an effective means of eliminating an intracellular bacterial niche and activating the host through release of inflammatory mediators such as IL-1 $\beta$, while sparing uninfected neighboring cells $(13,28-30)$. Thus, pyroptosis induced by inflammatory caspases $1 / 4 / 5 / 11$ is an innate immune response distinct from the canonical inflammasome activation pathway via cell-surface TLR4 (11, 31-34). However, caspase-11 can also be immunopathologic in sepsis (35). Caspase-11-deficient mice were protected in endotoxemic shock $(10,11)$, suggesting that in the setting of an overwhelming inflammatory response, the potentially protective pyroptotic mechanism activates an exaggerated pathologic response due to overwhelming cell lysis.

The role of the inflammatory caspases $4 / 5 / 11$ in mediating cytoplasmic LPS signaling and pyroptosis has until now been primarily studied in macrophages or dendritic cells $(11,12,14,28,32,36)$. Their role in destroying the endothelial barrier through widespread endothelial death and pathogenesis of ALI remains unknown. Here, we tested the hypothesis that lung ECs are a primary target for pyroptosis via intracellular sensing of LPS by the inflammatory caspases $4 / 5 / 11$ and that endothelial pyroptosis is required for the induction of ALI.

\section{Results}

LPS in ECs induces pyroptotic cell death via activation of inflammatory caspases. We first addressed the question of whether ECs sense LPS in the cytoplasm and initiate caspase-4/5/11-mediated pyroptosis. To mimic LPS internalization by uptake of bacterial microvesicles (23), we encapsulated FITC-labeled LPS in lipofectamine-based liposomes $(15,17)$ and transfected ECs. We observed the intracellular uptake of FITC-LPS by imaging flow cytometery (37) (Figure 1, A and B). This increased in a time-dependent manner so that half the endothelium displayed internalized LPS by 6 hours (Figure 1C). To assess in vivo uptake of LPS, FITC-LPS was injected i.p. in C57BL/6 mice. Here, we also observed the uptake of LPS in lung microvascular ECs (Supplemental Figure 1; supplemental material available online with this article; https:// doi.org/10.1172/JCI94495DS1). Using confocal microscopy, we generated a 3D Z stack image, which confirmed intracellular localization of systemically injected FITC-LPS in the lung endothelium (Supplemental Video 1). We next addressed whether intracellular LPS in ECs induced pyroptosis. LPS transfection caused massive EC lysis, whereas extracellular incubation with the same dose of LPS had no significant effect (Figure 1D). LPS transfection induced marked lactate dehydrogenase $(\mathrm{LDH})$ release $(\sim 40 \%)$, which was far greater than the cell lysis achieved by apoptosis (Figure 1E). 
A

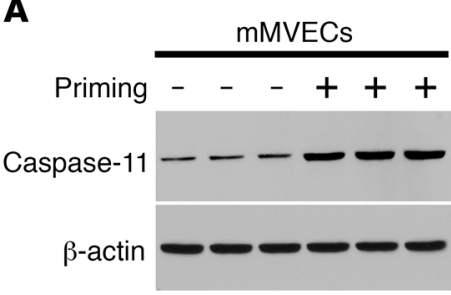

C

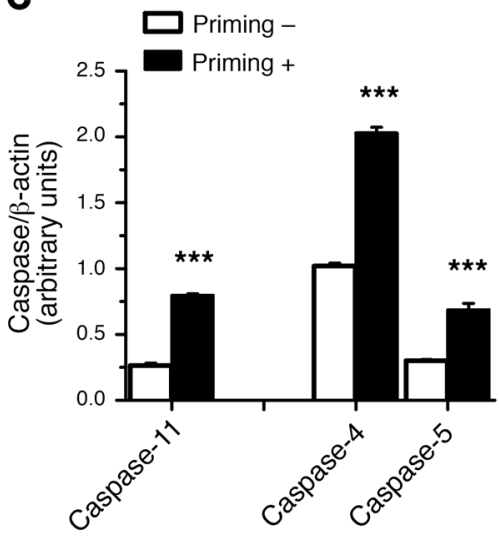

B

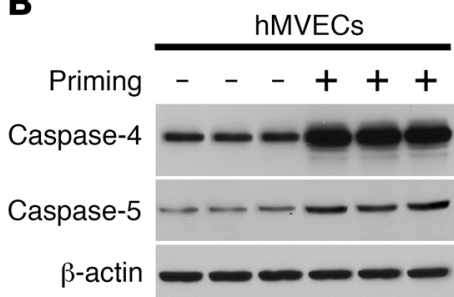

D

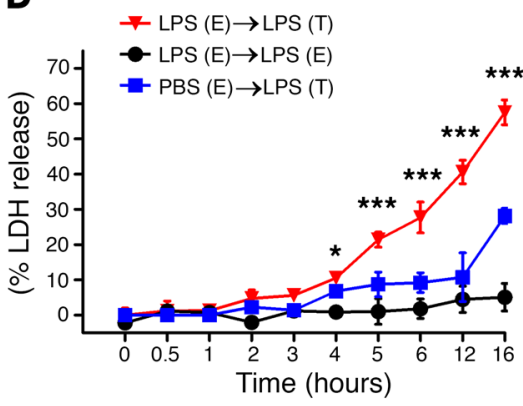

Figure 2. TLR4-mediated LPS signaling is required for activation of endothelial pyroptosis. Immunoblot analysis of the inflammatory caspase-11 in MMVECs (A) and the inflammatory caspases 4 and 5 in hMVECs (B) in the presence or absence of priming with extracellular $500 \mathrm{ng} / \mathrm{ml}$ LPS for 3 hours prior to transfecting the cells with $2 \mu \mathrm{g} / \mathrm{ml}$ LPS for 16 hours. (C) Quantification of the inflammatory caspase expression shows significant upregulation of caspases 4 and 5 in human ECs and caspase-11 in mouse ECs with priming. Statistics obtained from Student's 2-tailed $t$ test. (D) hMVECs were first primed with extracellular (E) LPS or PBS, then transfected (T) with LPS or only incubated (I) with extracellular LPS. (E) Dose dependence of EC lysis in hMVECs induced by intracellular LPS. (F) LDH release by mMVEC isolated from WT, $\mathrm{Tlr}^{-/ \text {, }}$, and Casp11/- mice after internalization of LPS for 16 hours. CTRL, control. (C) Western blot and (H) ELISA detection of mature IL-1 $\beta$ in hMVEC lysates and culture supernatants 16 hours after endothelial transfection with LPS $(2 \mu \mathrm{g} / \mathrm{ml})$ or without LPS transfection. (H) There was a significant amount of mature IL-1 $\beta$ release when ECs were primed with extracellular LPS and subsequently exposed to intracellular LPS for 16 hours. Results are shown as mean \pm SEM. $n \geq=5$. ${ }^{*} P<0.05 ;{ }^{* * *} P<0.001$. All statistics except C obtained from ANOVA.

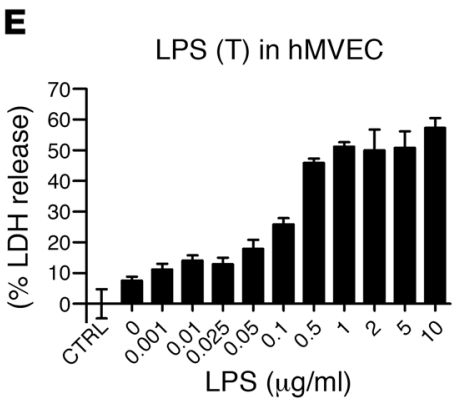

$\mathbf{F}$
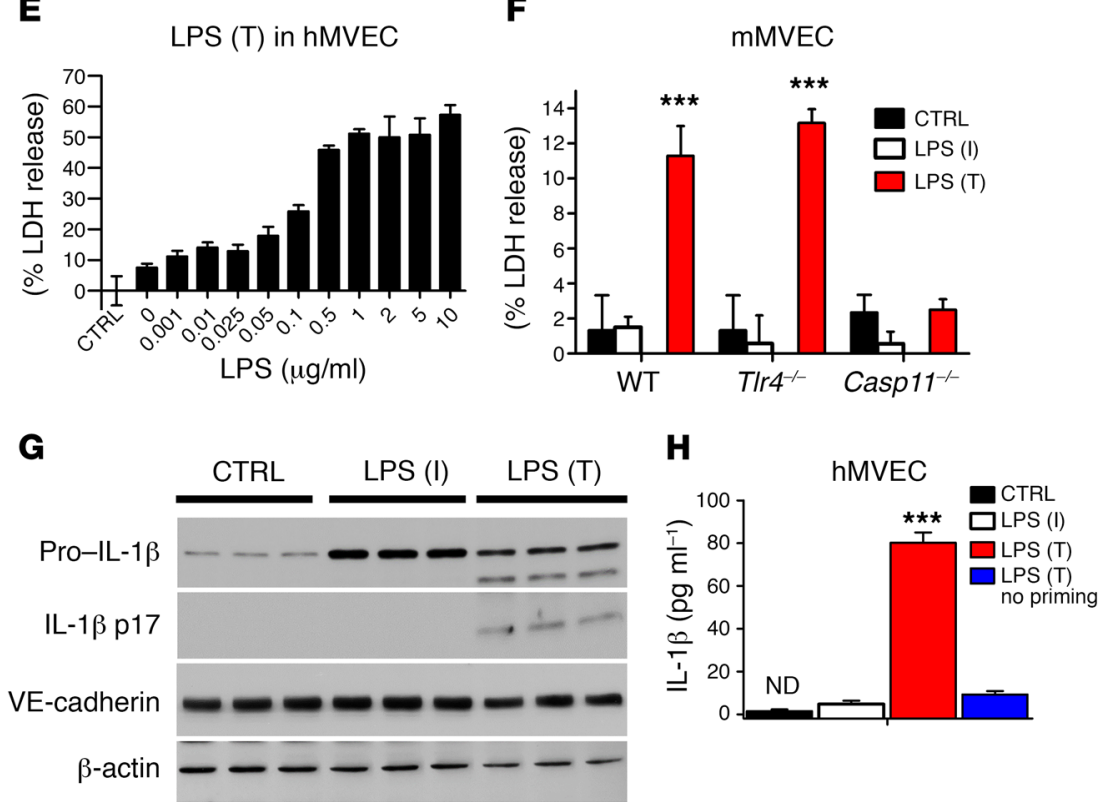

Cell lysis was blocked by the pan-caspase inhibitor z-VAD-FMK (Figure 1E), confirming that this was due to a caspasemediated lytic cell death. To identify the underlying mechanism, we investigated the role of the inflammatory caspases 4 and 5 in human ECs. Depletion of caspase- 4 or -5 by siRNAs prevented LPS-induced pyroptosis (Figure 1E). Further, LPS internalization triggered the pyroptosis response in all ECs studied, including human dermal microvascular endothelial cell line HMEC 1, human umbilical vein ECs (HUVECs), and mouse lung microvascular ECs (mMVECs-L) (Supplemental Figure 2A and Supplemental Figure 3, A and B).

TLR4-mediated LPS signaling synergizes with intracellular LPS to activate endothelial pyroptosis. We next addressed the role of a priming signal in ECs through stimulation with TLR agonists in activating pyroptosis. LPS priming via TLR4 induced expression of caspase-11 in murine ECs (Figure 2A). Human caspase- 4 is thought to be constitutively expressed in phagocytic cells (38), whereas human caspase-5 is inducible (39). We observed basal expression of caspase-4 and caspase-5 in human ECs, and both caspases were markedly upregulated in human ECs by priming with extracellular LPS (Figure 2B); the priming effect was more prominent with caspase-4 (Figure 2C). Primed ECs showed lysis as early as 3 hours after LPS transfection, whereas significant pyroptosis was observed in nonprimed cells only after 12 hours (Figure 2D), consistent with increased sensitivity of ECs due to priming- 
A

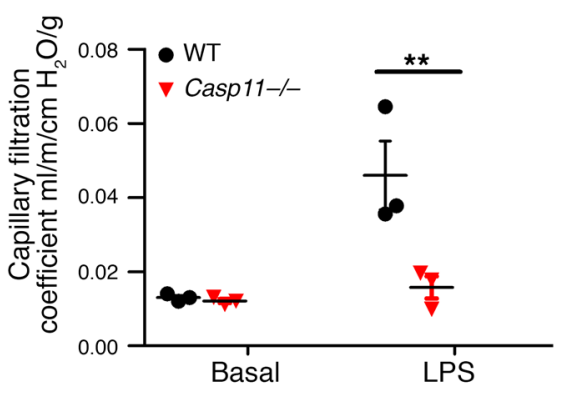

C

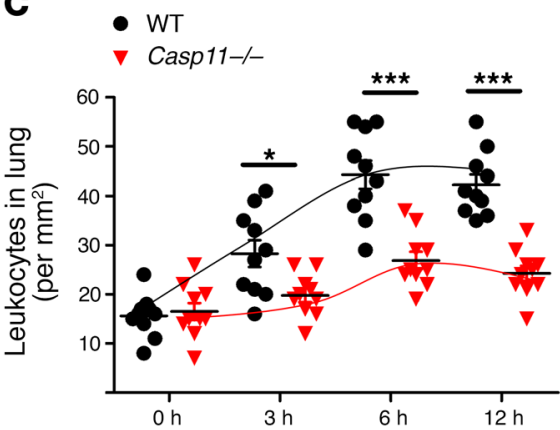

B

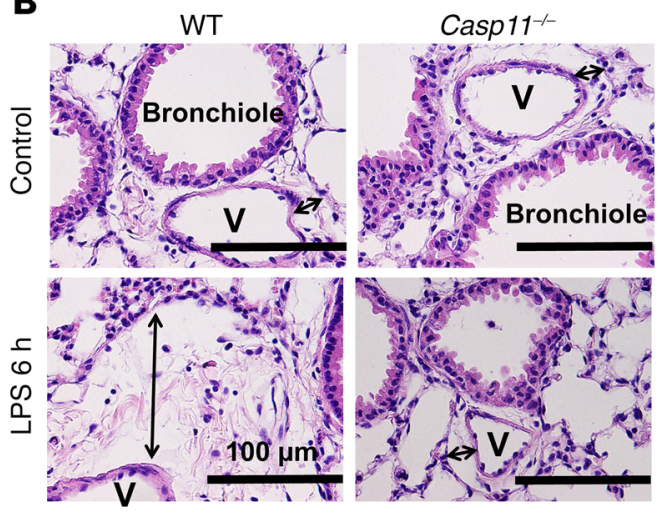

D

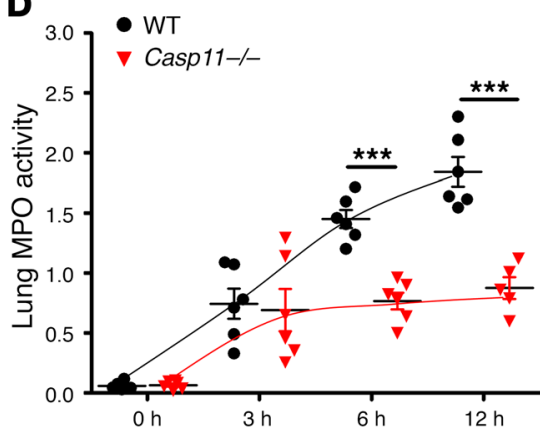

E

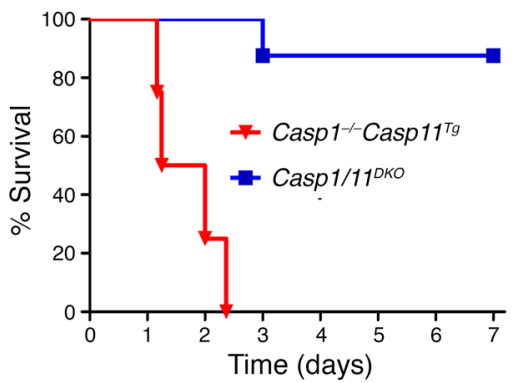

$\mathbf{F}$

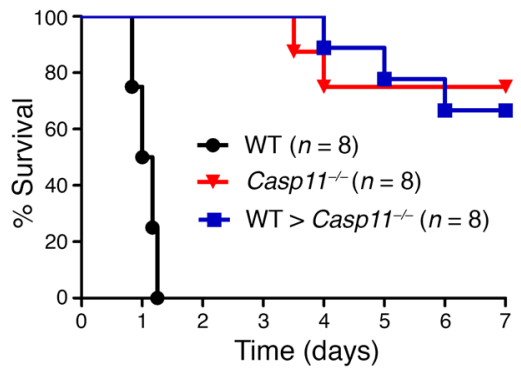

Figure 3. Requirement for caspase-11 expressed in nonhematopoietic cells in mediating endotoxemia-induced acute lung vascular injury and mortality. (A) Lung microvessel filtration coefficient (a measure of lung vascular permeability) was determined in WT and Casp11/-- mice, and the data points depicting individual mice are shown. Mice were exposed to systemic LPS (40 mg/kg i.p.) for 6 hours. Statistics obtained from 2-tailed Student's $t$ test.(B) H\&E-stained cross section of the lung from control mice and LPS-exposed mice at 6 hours shows interstitial edema (indicated by arrows) in WT but not in Casp11 $11^{-/-}$mice. Scale bars: $100 \mu \mathrm{m}$. V, lung microvasculature. Images are representative of 5 animals. Quantitative analysis for leukocyte infiltration in lungs (C) and lung tissue MPO activity (D). ${ }^{*} P<0.05$; ${ }^{*} P<0.01 ;{ }^{* *} P<0.001$. Statistics in C and $\mathbf{D}$ obtained from ANOVA. (E) Survival of Casp1/11 $1^{\mathrm{KKO}}$ mice and Casp1 ${ }^{-/-}$Casp11 $11^{\text {Tg }}$ mice subjected to a lethal dose of LPS (40 $\mathrm{mg} / \mathrm{kg}$ i.p.) was assessed and is presented as a Kaplan-Meier plot. (F) Casp11/- mice underwent BM irradiation and transplantation with WT BM to reconstitute caspase-11 in hematopoietic cells. Global Casp11/-- mice as well as Casp11/-mice transplanted with WT hematopoietic-lineage cell (BMT) chimeras were protected from lethal sepsis, while nontransplanted WT control mice were not. induced upregulation of inflammatory caspases. We also observed that LPS concentrations in primed ECs as low as 50 $\mathrm{ng} / \mathrm{ml}$ induced pyroptosis (Figure $2 \mathrm{E}$ ). To determine whether alternate priming pathways could substitute for TLR4, we also primed cells with TLR2 ligand $\mathrm{Pam}_{3} \mathrm{CSK}_{4}$ and TLR3 ligand poly(I:C). Here we found that priming with these TLR ligands also induced cell lysis similar to LPS (Supplemental Figure 2 and Supplemental Figure 3). As TLR4 may be dispensable for intracellular LPS-mediated pyroptosis in macrophages $(10,11)$, we isolated ECs from $\mathrm{Tlr}^{-/-}$mice and primed these cells with either $\mathrm{Pam}_{3} \mathrm{CSK}_{4}$ or poly(I:C) instead of LPS. These cells also responded to cytoplasmic LPS similarly to WT ECs (Figure 2F), whereas ECs generated from Casp11/- mice demonstrated no evidence of pyroptosis. Thus, caspase-11, but not TLR4, is required for intracellular LPS-mediated endothelial pyroptosis.

Caspases 4/5/11 activate NLRP3 inflammasome, resulting in IL-1 $\beta$ cleavage (31). We observed increased expression of pro-IL-1 $\beta$ after priming, while maturation required cytoplasmic LPS in ECs (Figure 2G). There was minimal release of mature IL-1 $\beta$ with extracellular LPS incubation or with intracellular
LPS in the absence of priming, but there was marked mature IL-1 $\beta$ release when ECs were primed with extracellular LPS and subsequently exposed to cytoplasmic LPS (Figure $2 \mathrm{H}$ ). Thus, priming of ECs through upregulation of caspases 4/5/11 markedly enhanced IL-1 $\beta$ production, maturation, and secretion and the full activation of pyroptosis.

Caspase-11 is required for endotoxemia-induced lung vascular hyperpermeability and mortality. To evaluate the role of caspase-11 in the pathogenesis of ALI-induced endotoxemia, we first determined lung microvessel filtration coefficient (as a measure of lung vascular permeability) in WT and Casp11 $11^{-/}$mice. At 6 hours after exposure to a lethal dose of systemic LPS (40 mg/kg i.p.), WT mice showed a marked increase in lung vascular leakiness that is characteristic of ALI, while the response was abrogated in Casp11/mice (Figure 3A). Cross sections of lung showed edema in WT mouse lungs, whereas Casp11/- mice were protected (Figure 3B). Analysis for leukocyte accumulation (Figure 3C) and myeloperoxidase (MPO) activity (Figure 3D) (used as a marker for neutrophil influx) demonstrated that caspase-11 was required for leukocyte infiltration in endotoxemia-induced ALI. 
A

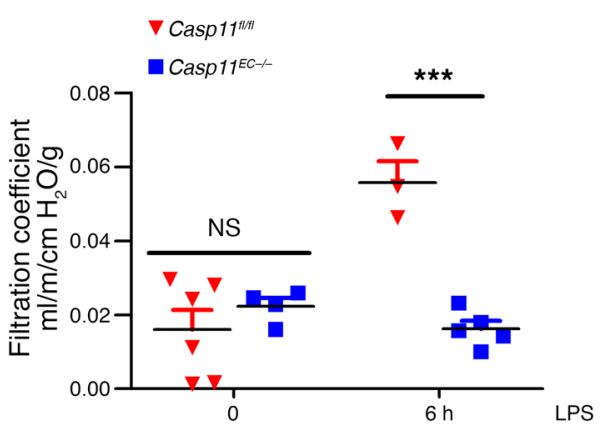

C

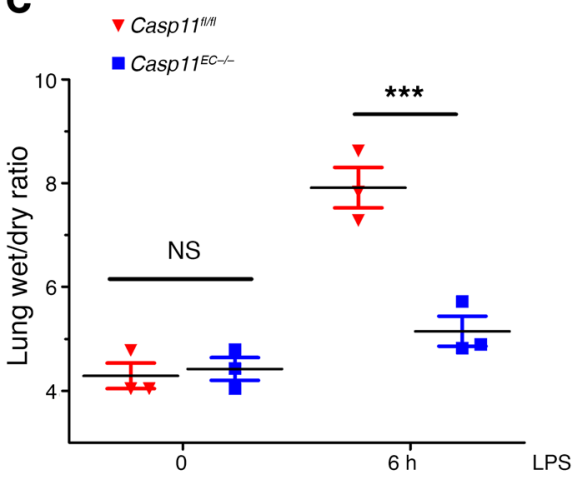

E

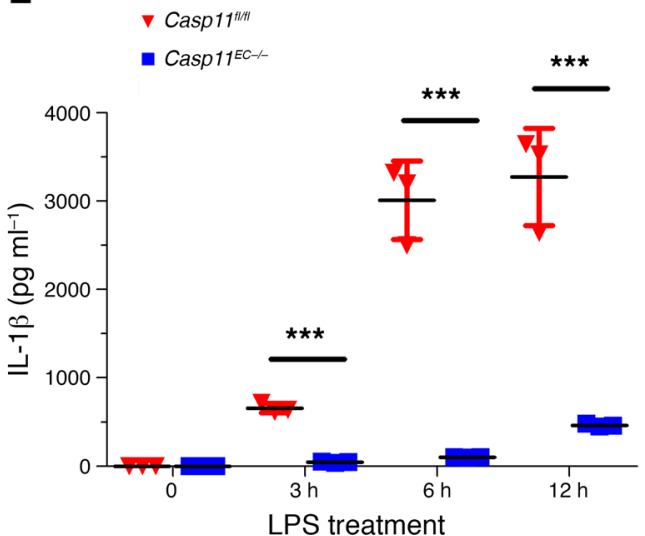

D
B
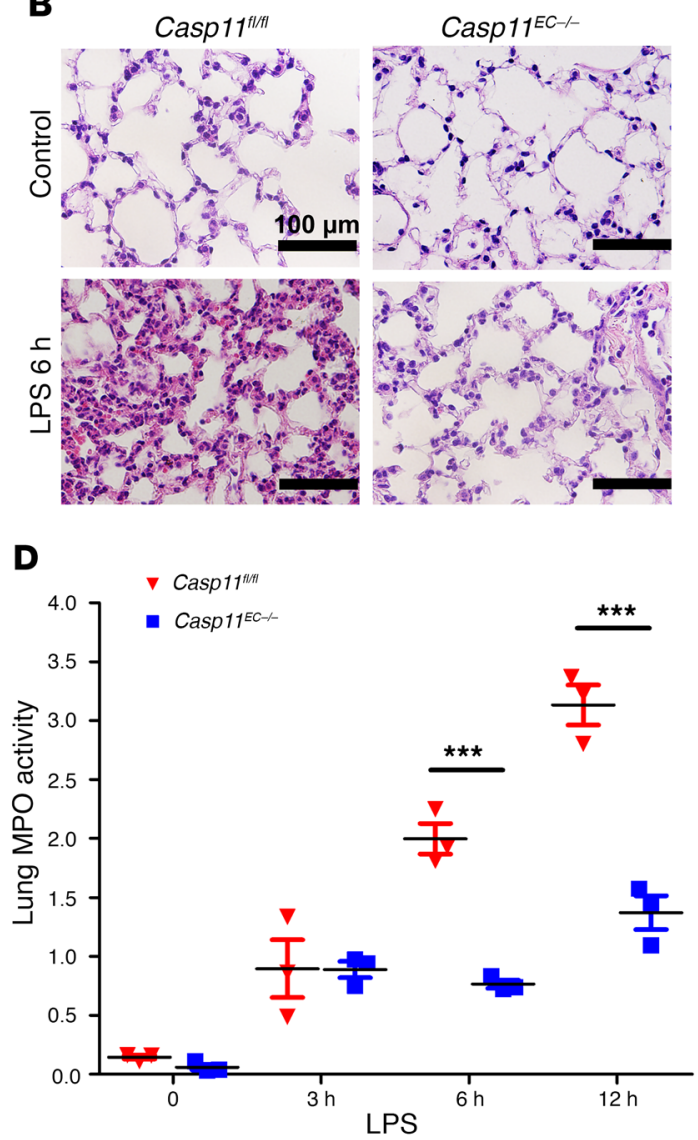

$\mathbf{F}$

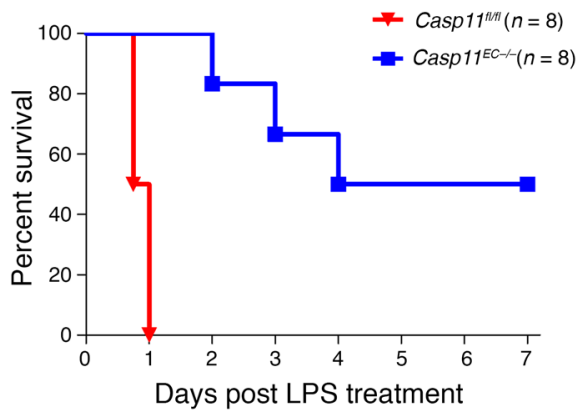

Figure 4. EC-expressed caspase-11 Is required for $A L I$ induced by endotoxemia. (A) Lung microvessel filtration coefficient was assessed in Casp1 $17^{f / f l}$ and Casp $11^{\mathrm{E} C-/-}$ mice following exposure to systemic LPS ( $40 \mathrm{mg} / \mathrm{kg}$ i.p.) for 6 hours. (B) Representative H\&E staining ( $n=6$ mice per group) of lung sections from Casp17 $11^{\mathrm{flfl}}$ and Cas $11^{\mathrm{EC}-\mathrm{C}}$ mice shows marked reduction in inflammation and lung injury in the latter group at 6 hours following LPS (40 mg/kg i.p.). Scale bars: $100 \mu \mathrm{m}$.

(C) A similar protective effect was also observed when assessing the lung wet/dry ratio. (D) Quantitative analysis for neutrophil infiltration in the lungs by assessing lung tissue MPO activity. (E) Circulating levels of the proinflammatory cytokine IL-1 $\beta$, which is released during pyroptosis. Scatter plots show mean \pm SEM. Dots represent data from individual mice. ${ }^{* *} P<0.001$. (F) Kaplan-Meier survival plots of mice challenged with a lethal LPS dose $(40 \mathrm{mg} / \mathrm{kg}$ i.p.) show that EC-specific deletion of caspase-11 improves survival from $0 \%$ to $50 \%-60 \%$. Statistics obtained from 2-tailed Student's $t$ test.
To differentiate between the roles of inflammatory caspase- 1 and caspase-11 in inducing mortality, survival of Casp1 $1^{-/-}$Casp1 $^{1--}$ (Casp1/11 ${ }^{D K O}$ ) mice and Casp1 $1^{-/-}$Casp11 ${ }^{T g}$ mice was studied following exposure to a lethal dose of LPS (40 mg/kg i.p.). Genetic deletion of both inflammatory caspases resulted in $90 \%$ survival, whereas mice lacking caspase-1 but expressing caspase-11 exhibited 0\% survival within 72 hours (Figure 3E), indicating the central role of caspase-11 in the mechanism of endotoxemia-induced death.

We also determined whether the protective effect of caspase-11 deletion was due to caspase-11 expressed in hematopoietic versus nonhematopoietic cells. Casp11 $11^{-/-}$mice underwent BM irradiation and transplantation with WT BM to reconstitute WT caspase-11 in

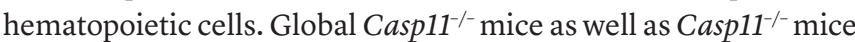
transplanted with WT hematopoietic-lineage cells were protected from lethal sepsis, while control WT mice were not (Figure 3F), thus demonstrating the crucial role of caspase- 11 expressed in nonhematopoietic cells for endotoxemic mortality.

We also investigated whether caspase-11 is important in the mechanism of ALI and mortality induced by polymicrobial sepsis in the cecal ligation puncture (CLP) model. Lung sections from Casp11 $1 /$ mice showed reductions in inflammation and lung injury compared with those from control mice after CLP for 12 hours (Supplemental Figure 4A). Deletion of caspase-11 prevented CLP-induced mortality (Supplemental Figure 4B).

Endothelial-expressed caspase-11 is required for endotoxin-induced $A L I$. We next investigated whether ALI induced by endotoxin was due to caspase-11 expressed in the endothelium per se. Here, we used mice carrying a LoxP site-flanked Casp11 for tis- 
A
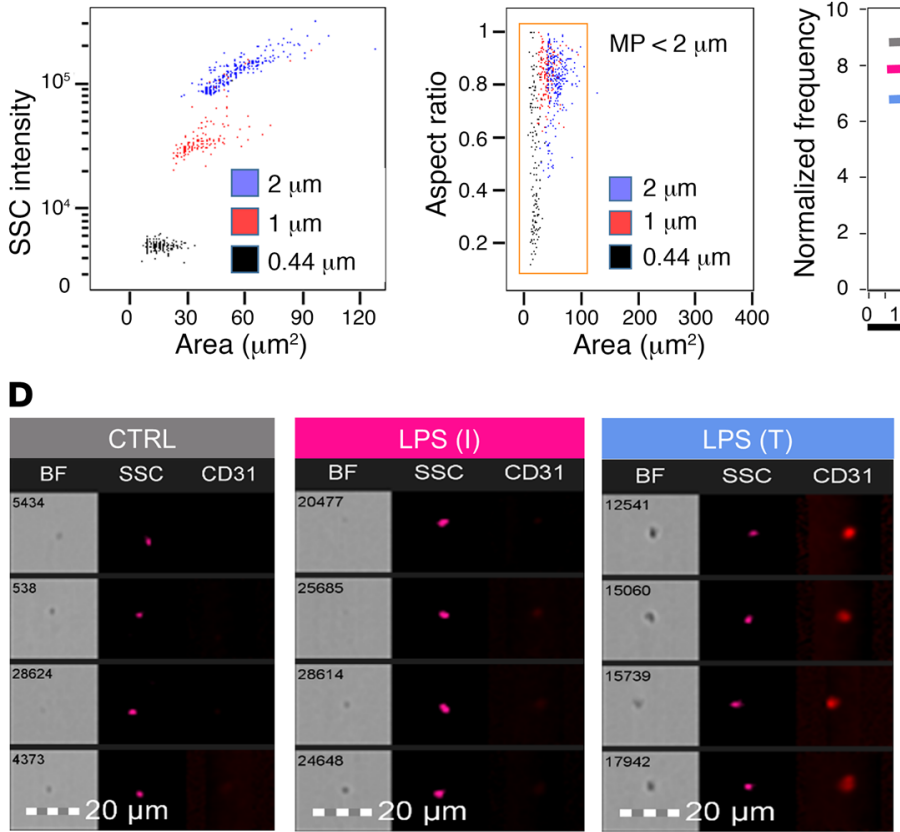

E
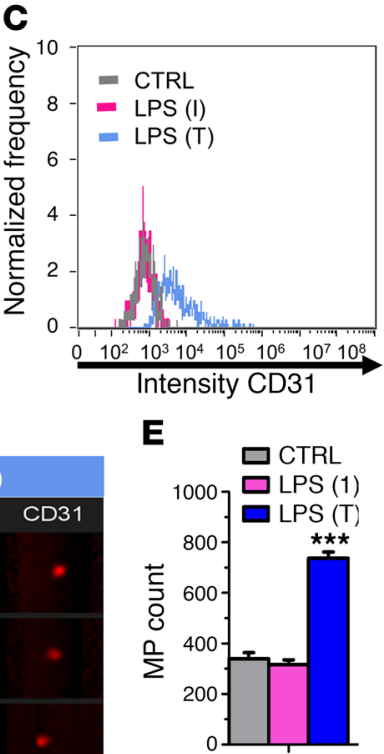

Intensity CD31

E

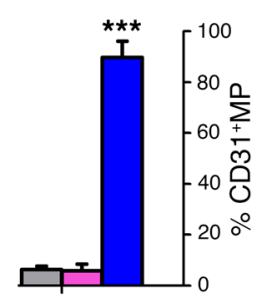

$\mathbf{F}$

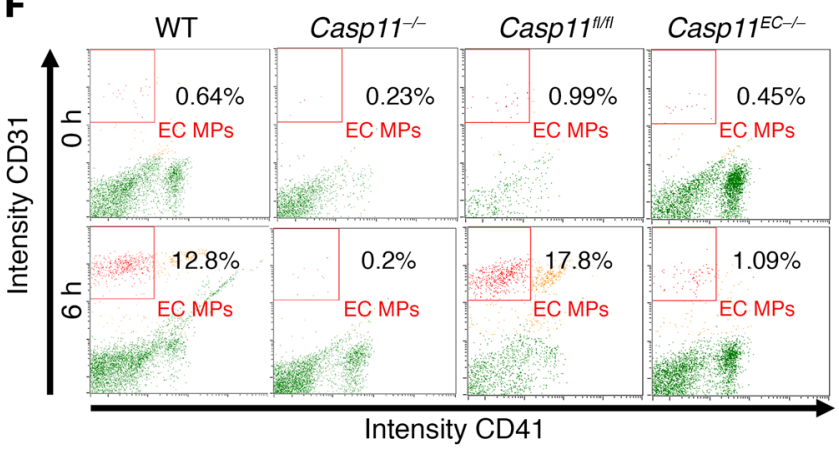

G

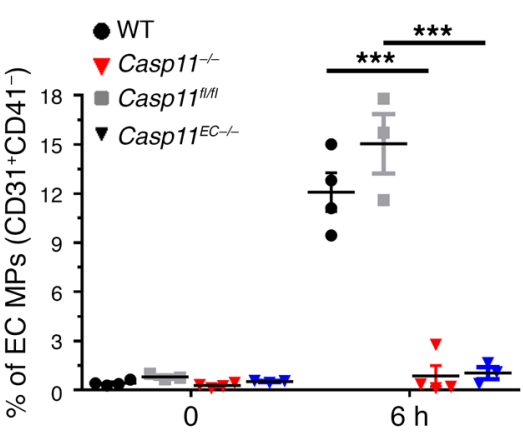

I

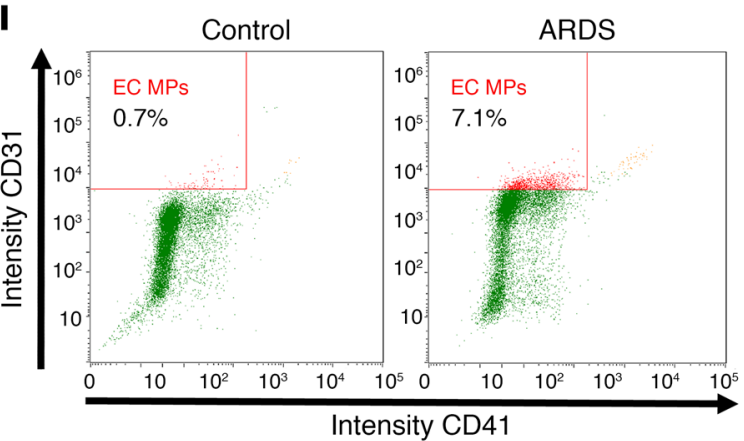

H

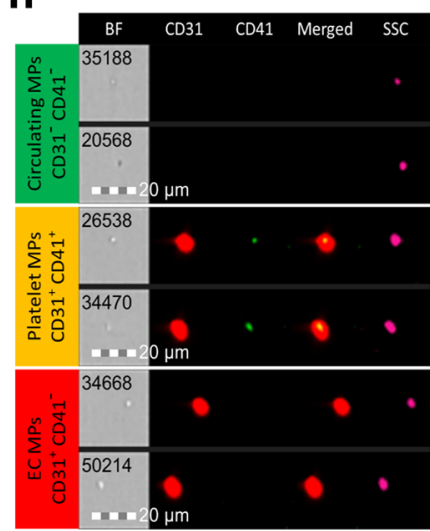

J

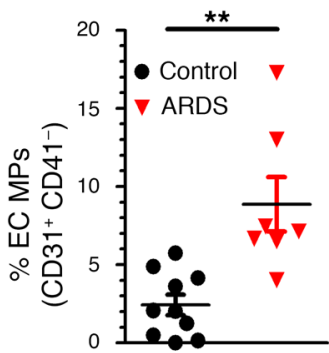

Figure 5. Pyroptotic ECs generate MP in caspase-11-dependent manner. (A and B) Flow cytometry showing size calibration and gate definition of MPs using fluorescent polystyrene bead standards of various sizes. MPs were gated by size gating $(<2 \mu \mathrm{m})$ correlated with object area in the bright field channel and intensity of the dark field side scatter (SSC). (C) Pyroptotic ECs released CD31+ MPs. Supernatants were collected from either control hMVECs only incubated (I) with LPS or primed cells transfected with LPS $(2 \mu \mathrm{g} / \mathrm{ml})$ for 16 hours. Samples were then analyzed using imaging flow cytometer. (D) Representative images of MPs from control (gray), LPS incubation (magenta), and LPS transduction (T) (blue). (E) Pyroptotic ECs displayed increase in total MP counts as well as enhanced CD31+ expression on MPs. Representative flow cytometry dot blots (F) and quantification (G) of MPs measured in the plasma of WT, Casp11-1-,

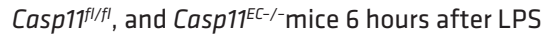
challenge (40 mg/kg i.p.). Endothelial-specific deletion of caspase-11 prevented the generation of endothelial MPs similarly to global caspase-11 deletion, thus demonstrating that EC MP release was due to the activation of endothelial caspase-11. (H) Micrograph of MPs from mouse plasma, visualized by imaging flow cytometry, showing MPs of endothelial (CD31+CD41-) and platelet (CD31+CD41 $\left.{ }^{+}\right)$ origins. Assessment of endothelial-derived MPs from plasma of healthy volunteers (control) and ARDS patients (described in Supplemental Table 1) in a representative flow cytometry dot plot (I) and a bar graph quantification (J) shows significant increases in endothelial MP release, consistent with endothelial pyroptosis during ALI/ARDS in patients. ${ }^{* *} P<0.01$; ${ }^{* *} P<0.001$ by ANOVA (E and $\mathbf{C}$ ) and 2-tailed Student's $t$ test (J). 
sue-specific deletion of caspase-11 and induced endothelial-

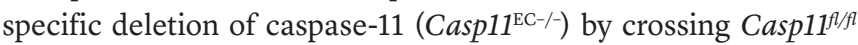
mice with Endo-SCL-Cre mice (40), which express the Crerecombinase in the adult endothelium upon induction with tamoxifen. Lung microvessel filtration coefficients determined in Casp11 $11^{\mathrm{EC}-/-}$ mice following exposure to systemic LPS (40 mg/ $\mathrm{kg}$ i.p.) demonstrated that EC-specific deletion of caspase-11 did not affect baseline lung vascular leakiness, but fully prevented LPS-induced lung vascular leakiness in contrast with Casp11 $1 / f / f$ mice (Figure 4A). Lung sections from Casp11 $11^{\mathrm{EC}-/-}$ mice showed marked reduction in inflammation and lung injury at 6 hours following LPS (40 mg/kg i.p.) as compared with sections from Casp11 $1 / f l$ mice (Figure 4B). Endothelial-specific caspase-11 deletion also prevented the increase in lung wet/dry weight ratio, a measure of tissue edema (Figure 4C). Further, analysis for neutrophil infiltration (Figure 4D) and circulating levels of IL-1 $\beta$, released during pyroptosis, also showed marked reductions following EC-specific caspase-11 deletion, which was comparable to protection seen in mice with global caspase-11 deletion (Figure $4 \mathrm{E})$. Kaplan-Meier survival plots of mice challenged with a lethal LPS dose (40 mg/kg i.p.) demonstrated that EC-specific deletion of caspase- 11 improved survival from $0 \%$ to $50 \%-60 \%$, whereas global caspase-11 deletion increased survival to $70 \%-80 \%$ at 7 days (Figure 4F). Similar results were seen in lung microvessel filtration coefficient (Supplemental Figure 5A) and KaplanMeier survival analysis (Supplemental Figure 5B) of Casp11 $1^{\mathrm{EC}-}-$ mice challenged with CLP for 12 hours, thus demonstrating the central role of endothelial caspase-11 in mediating ALI and mortality in polymicrobial sepsis.

Pyroptotic ECs generate microparticles in a caspase-11-dependent manner. It remains unclear whether EC-derived microparticles (MPs) are generated during caspase-11-dependent pyroptosis in incipient ALI. Here, we used imaging flow cytometry to evaluate MP generation from pyroptotic ECs. MPs were identified by size gating $(<2 \mu \mathrm{m})$ correlated with object area in the bright field channel and intensity of the dark field side scatter (Figure 5, A and B). We observed that ECs released MPs in vitro following LPS transfection, but not LPS incubation (Figure 5C), indicating that cytoplasmic LPS entry was required for MP release. Pyroptotic ECs displayed increases in total MP counts, and percentages of MPs positive for the endothelial surface marker PECAM (CD31+) increased to $90 \%$ (Figure 5, D and E), indicating that fragmented endothelial plasma membrane was the primary source of MPs released by cytoplasmic LPS challenge.

We next examined MP generation in vivo during endotoxemia. EC MPs were assessed by labeling for CD31, while the platelet marker CD41 was used to exclude platelet MPs, which are also positive for CD31. Representative flow cytometry dot blots (Figure 5F) and quantification (Figure 5G) of circulating MPs in WT, Casp11/-, Casp11 $1^{f l / f l}$, and Casp11 ${ }^{E C-/}$ mice 6 hours after LPS challenge (40 mg/ kgi.p.) demonstrated that endothelial-specific deletion of caspase-11 prevented the generation of endothelial MPs. This response was similar to that observed with global caspase-11 deletion, indicating that EC MP release was due to activation of endothelial caspase-11. Micrographs of MPs in mouse plasma showed that the majority of circulating MPs in endotoxemia were derived from ECs (Figure $5 \mathrm{H})$. To address relevance to ARDS, we determined endothelial
MP levels in the plasma of healthy volunteers and ARDS patients (described in Supplemental Table 1). Flow cytometry dot plot (Figure 5I) and quantification (Figure 5J) showed significantly greater release of endothelial MPs in ALI/ARDS patients, consistent with the results in mice above. To assess whether the increase in the circulating endothelial MP count was also seen in other forms of pulmonary edema, we quantified endothelial MPs in patients with cardiogenic pulmonary edema (CPE). No significant difference was observed in the circulating MP levels of CPE patients when compared with control subjects(Supplemental Figure 6), demonstrating the relevance of the circulating endothelial MP count as an indicator of widespread endothelial destruction during endotoxemia.

Endothelial caspase-11 activation is required for mature $I L-1 \beta$ generation and Gsdmd cleavage. IL-1 $\beta$ is a proinflammatory cytokine synthesized as a $31-\mathrm{kD}$ a precursor protein and proteolytically activated by inflammatory caspases to release mature IL-1 $\beta$ (also known as p17) protein during pyroptotic cell death $(12-14,18,21)$. To address whether IL-1 $\beta$ cleavage is mediated by EC-specific

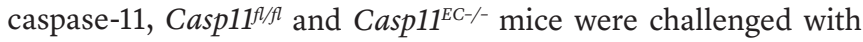
LPS (40 mg/kg i.p.). Lung ECs were isolated and immunoblotting was performed for pro-IL-1 $\beta$ and mature IL-1 $\beta$ protein. ECspecific deletion of caspase-11 prevented pro-IL-1 $\beta$ generation following endotoxemia (Figure 6, A and B). Another critical target of caspase-11 is the pore-forming pyroptosis perforin Gsdmd, which is cleaved into its active form by caspase- 11 and forms pores promoting cell swelling and lytic cell death $(12,14-17)$. We observed that cytoplasmic LPS in human ECs markedly increased formation of the active, cleaved Gsdmd p30 protein (Figures 6, C and D). Moreover Gsdmd cleavage was inhibited in lung ECs of Casp11 $11^{E C-/}$ mice following LPS challenge (Figure 6, E and F).

\section{Discussion}

Pyroptosis of phagocytes mediated by inflammatory caspases plays an important role in innate immune defense against intracellular bacteria and endotoxin $(13,28-30,41)$. It is characterized by lytic cell death induced by the binding of intracellular LPS to caspases $4 / 5$ in human cells and caspase-11 in mouse cells, which in turn leads to Gsdmd-induced pore formation and cleavage of inflammatory procytokine IL-1 $\beta$, unlike other forms of programmed cell death (25). Thus, pyroptosis strikes a precarious balance between activating the host-defense response and minimizing collateral tissue damage $(10,21)$. As unfettered pyroptosis can induce tissue injury, targeting caspase-activated pyroptosis in specific cell types may be a useful strategy for limiting inflammatory tissue damage and organ failure without compromising host defense. The present study investigated the role of pyroptosis specifically in ECs and the potential of targeting endothelial pyroptosis in ALI. ECs demonstrated lytic cell death, activation of Gsdmd, and release of the proinflammatory cytokine IL-1 $\beta$, processes that are dependent on caspase- $4 / 5$ in human ECs and caspase- 11 in mouse ECs. We demonstrated on the basis of genetic caspase-11 deletion studies in mice the requisite role of endothelial pyroptosis in the mechanism of LPS-induced ALI. We showed that LPS sensing in ECs via caspase-11 in mice mediated pyroptosis and hence was required for the development of ALI. LPS entered endothelial cytoplasm via bacterial microvesicles or bacterial breaching of the endothelial plasma membrane, and intracellular LPS then 
A

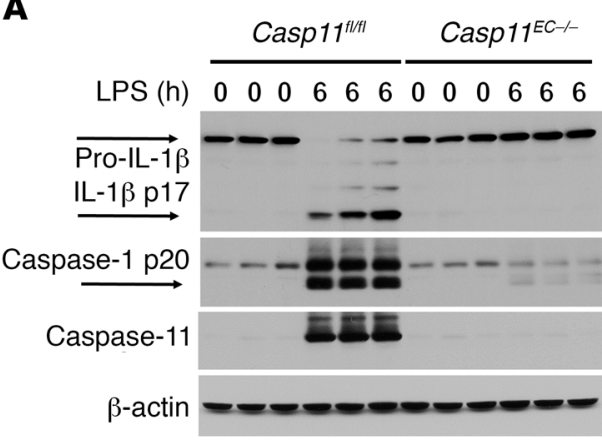

B

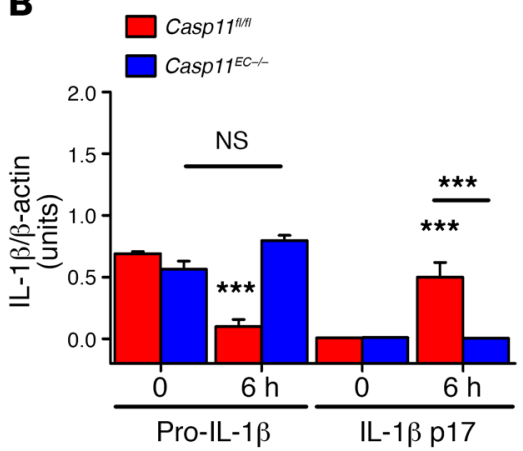

C

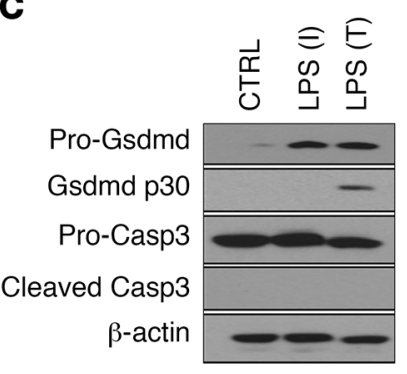

D

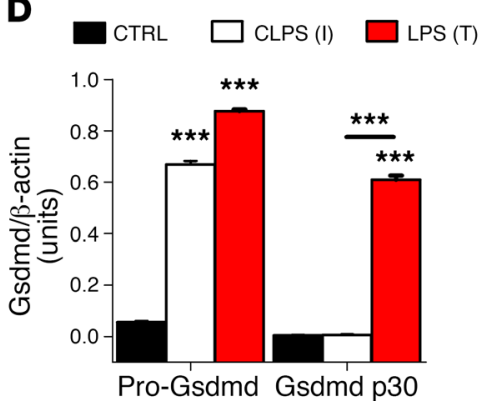

Figure 6. Endothelial caspase-11 activation is required for generation of mature IL-1 $\beta$

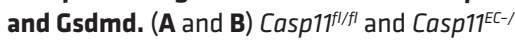
mice $(n=3)$ were challenged with LPS (40 $\mathrm{mg} / \mathrm{kg}$ i.p.) for 6 hours. ECs were isolated from lungs, and immunoblot analysis was performed for pro-IL-1 $\beta$, mature IL-1 $\beta$, and caspase- 1 cleavage, as shown in representative immunoblots and the bar graph quantification. (C and $\mathbf{D}$ ) Immunoblot analysis of the pore-forming mediator of pyroptosis Gsdmd showed that LPS $(2 \mu \mathrm{g} / \mathrm{ml})$ transfection markedly increased the formation of the active, cleaved Gsdmd p30 protein in human ECs. (E and F) Gsdmd cleavage was suppressed in ECs of Casp $11^{-/-}$mice $(n=3)$ following LPS challenge for 6 hours. ${ }^{* *} P<0.001$ versus control or as indicated. Data are shown as mean \pm SEM. Statistics obtained from ANOVA.

$\mathbf{E}$

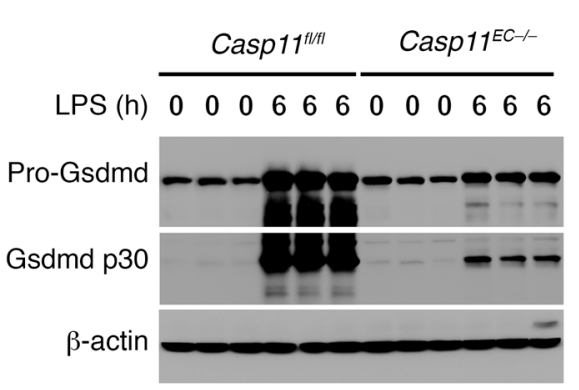

$\mathbf{F}$

F $\square$ Casp $11^{1 / \mathrm{NA}}$

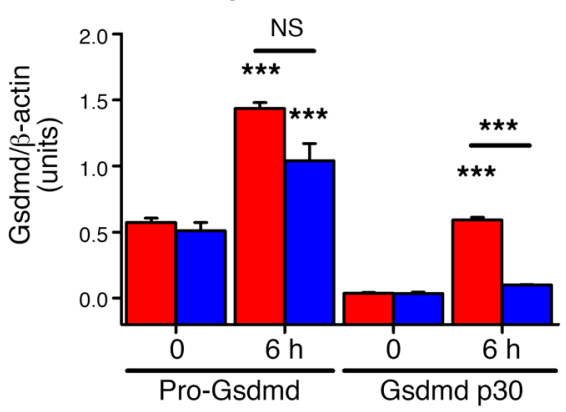

induced caspase- $4 / 5 / 11$ activation, which triggered pyroptosis via Gsdmd cleavage (Figure 7). We demonstrated that widespread lysis of ECs resulted in severe disruption of the endothelial barri$\mathrm{er}$, release of proinflammatory cytokines, influx of leukocytes, and lung edemagenesis, all hallmarks of ALI $(2,42,43)$.

Caspase-11-dependent pyroptosis triggered by intracellular LPS was described in macrophages and dendritic cells (10-12, $14,16,22,30,36)$; however, the critical role of this noncanonical intracellular LPS sensing mechanism in ECs has not been established. Here, we demonstrated a highly sensitive and intricate intracellular LPS-sensing machinery in ECs, which resulted in caspase-4/5/11-dependent endothelial lysis. Distinctive features included rupture of the plasma membrane and release of LDH, and maturation and release of IL-1 $\beta$ as well as cleavage of Gsdmd, the pyroptosis effector perforin $(12,14,15,17)$. We demonstrated that endothelial-specific deletion of caspase-11 in 2 distinct models of ALI, endotoxemia and the CLP model of polymicrobial sepsis, prevented LPS-induced increases in lung vascular endothelial permeability and markedly enhanced survival. BM transplantation of WT hematopoietic cells in mice with global caspase-11 deficiency abrogated endotoxemia-induced
ALI, establishing the central role of caspase-11 expressed in ECs in the mechanism of ALI.

Priming with inflammatory activators, such as LPS, poly(I:C), IFN- $\beta$, and IFN- $\gamma$, increases the expression of inflammasome components, which in turn are required for activation of the noncanonical inflammasome pathway responsible for pyroptosis induction in phagocytic cells $(10,11)$. Priming with extracellular LPS occurred via LPS binding to TLR4 and downstream TLR4 signaling, whereas pyroptosis induction was mediated via a TLR4independent mechanism, since intracellular LPS induced pyroptosis by directly binding to the intracellular caspases. We demonstrated that priming by extracellular LPS enhanced the expression of caspase- 4 and caspase- 5 in human ECs and caspase-11 in mouse ECs, thereby augmenting the subsequent pyroptotic response induced by intracellular LPS binding to these inflammatory caspases. Although ECs in the absence of priming can also undergo pyroptosis, this requires prolonged exposure to LPS. Thus, the threshold for LPS-induced pyroptosis in the endothelium set by expression of inflammatory caspases may serve to prevent undue activation of the potentially harmful pyroptotic pathway in settings of mild or transient endotoxemia. 
Intracellular LPS sensing in ECs also highlights its role as an immune cell and regulator of innate immunity. ECs line the entire vasculature and are thus the first point of contact for circulating bacterial toxins and pathogens. A responsive endothelialbased LPS detection system thus provides a defense mechanism to activate host defense at the blood-tissue interface. In a 2-tiered hierarchy of immune surveillance proposed by Iwasaki and Medzhitov (44), the EC should be included not only as an "effector cell" regulating influx of inflammatory cells and fluid and plasma proteins into tissue, but also as a cell capable of sensing intracellular LPS and activating pyroptosis.

An important translational finding was that ECs undergoing pyroptosis released MPs. MPs derived from ECs were reported to be elevated in sepsis (45) as well as other forms of vascular injury (46-48), but their link to endothelial pyroptosis is not known. Our findings show that endothelial MPs were increased in endotoxemic WT mice but not in Casp11/-- mice. In addition, we observed increased endothelial MPs in plasma from ARDS patients when compared with healthy subjects, suggesting that circulating MP levels could be a biomarker for endothelial pyroptosis in ALI/ ARDS patients and help stratify patients who may benefit from therapeutic interventions to contain endothelial pyroptosis. Conventional flow cytometry represents the mainstay of MP measurements, yet application toward characterization of submicrometer MPs remains a challenge due to low sensitivity and accuracy. Imaging flow cytometry allowed us to interrogate each event that passes through the flow cell, pinpointing the fluorescent signals and allowing for the detection of MP populations (49). Our studies suggest that endothelial MPs serve as a biomarker for caspase-11mediated endothelial pyroptosis, but they also raise the intriguing question of whether MPs themselves can initiate signaling events that perpetuate or amplify endothelial injury and pyroptosis.

Gsdmd has been identified through genetic screening as a key mediator of pore formation of cells undergoing pyroptosis $(12,14-18)$. Gsdmd is a critical target of caspase-11 (19), which cleaves it to generate N-terminal (NT) fragments, which are the "executioners" of pyroptosis $(12,14)$. Overexpression of cleaved Gsdmd-NT alone induced pyroptosis independently of caspase-11 in vitro (14). Also, BM-derived macrophages (BMDMs) lacking functional Gsdmd were resistant to death in response to intracellular LPS, whereas deletion of inflammasome components or caspase-1 continued to exhibit Gsdmd cleavage $(12,14)$. However, the interrelationship of caspase- 1 and caspase- 11 and the mechanisms involved in endothelial pyroptosis and related ALI remain unclear. Endothelial-specific caspase-11 depletion prevented LPS-induced caspase-1 cleavage, but had no effect on the basal caspase- 1 expression. Thus, studies are needed to clarify overlapping roles of caspase- 1 and caspase- 11 in endothelial pyroptosis. Even though our studies establish an essential role for endothelial caspase-11 in mediating pyroptosis of the endothelium and ALI, it remains unknown whether this process also activates the endothelial inflammasome in a noncanonical manner similar to that reported for phagocytic cells $(12,14)$.

In summary, we demonstrate that pyroptosis of ECs has a fundamental homeostatic role in host defense and immune surveillance functions of the microvasculature. Increased expression of caspase- $4 / 5$ in human ECs and LPS breaching of the endothelial

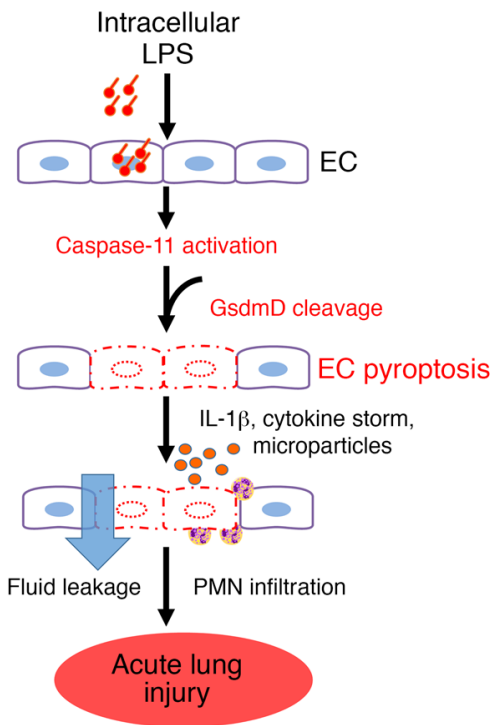

Figure 7. Model of EC caspase-11-dependent mechanism of endothelial pyroptosis and ALI. LPS enters the endothelial cytoplasma via bacterial microvesicles or by bacterial breaching of the EC plasma membrane. Intracellular LPS then triggers caspase-11-dependent EC pyroptosis and disrupts the endothelial barrier, resulting in pulmonary edema, release of proinflammatory cytokines, fluid protein leakage, and massive influx of leukocytes. PMN, polymorphonuclear leukocytes.

plasma membrane in sepsis are required for activation of endothelial pyroptosis; they thereby contribute to the development of fulminant pulmonary edema and ALI. Thus, therapeutic strategies aimed at preventing endothelial pyroptosis may preserve lung endothelial integrity and be beneficial in treating ALI/ARDS.

\section{Methods}

Mice. Mice were bred and maintained under specific pathogen-free conditions at the University of Illinois at Chicago animal facility. All mice were randomly assigned to experimental animal groups. Casp11 $11^{-1}$,

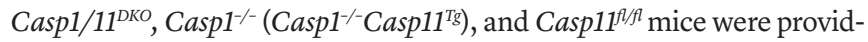
ed by Vishva Dixit (Genentech) (12). EC-specific caspase-11-knockout

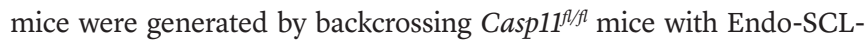
Cre mice, which express an endothelial-specific Cre in the adult (40). C57BL/6J mice initially acquired from The Jackson Laboratory and bred at the University of Illinois at Chicago were used as WT controls. Male or female mice 8 to 12 weeks old were used for all experiments.

Materials. Human lung microvascular ECs (hMVECs), endothelial growth medium 2 (EBM-2), and a nucleofector kit and electroporation system were obtained from Lonza. FuGENE transfection reagent was procured from Promega. Anti-Alexa Fluor 568 and 488 antibodies and ProLong Gold Antifade were obtained from Invitrogen. Mouse antirabbit IgG (conformation specific) antibodies were purchased from Cell Signaling Technology. Ultra-pure LPS (E. coli O111:B4), Pam3CSK4, and poly(I:C) LMW were obtained from InvivoGen. We obtained antibodies targeting caspase-1 (AdipoGen, AG-20B-0042-C100), IL-1 (R\&D Systems, AF-401-NA), caspase-11 (Novus Biologicals, NB-120-10454), caspase-4 (Santa Cruz Biotechnology Inc., sc-56056), caspase-5 (Santa Cruz Biotechnology Inc., sc-393346), $\beta$-actin (Santa Cruz Biotechnology Inc., sc-47778), GAPDH (Abcam, ab-8245), 
VE-cadherin (Santa Cruz Biotechnology Inc., sc-6458), and Gsdmd (Santa Cruz Biotechnology Inc., sc-393656). Anti-human CD31-APC and CD41-FITC antibodies were purchased from BD Bioscience.

EC culture. mLMVECs-L were isolated as previously described. In brief, blood-free mouse lungs were minced, digested with collagenase at $37^{\circ} \mathrm{C}$ for 45 minutes, triturated, and centrifuged at $1,000 \mathrm{~g}$. Cell suspension was incubated with PECAM-1-coated Dynabeads for 1 hour, after which ECs were magnetically sorted. Isolated ECs were plated on fibronectin-coated T-25 flasks and cultured with DME containing endothelial growth supplement. Cells were trypsinized and characterized by FACS analysis using anti-VE-cadherin, anti-VEGFR2, and anti-CD31 antibodies, which are known EC surface markers. We also determined tube formation in Matrigel as additional indices of EC characteristics. Isolated MLMVECs were more than 90\% pure.

Human pulmonary artery endothelial cells (HPAECs) were cultured in a T-75 flask coated with gelatin and growth factorsupplemented EGM2 medium, as previously described. hMVECs were cultured in EBM-2 supplemented with 10\% endotoxin-free fetal bovine serum (Omega Scientific). Adherent hMVECs were then cultured overnight in 96 -well plates at $1 \times 10^{6}$ cells $/ \mathrm{ml}$ before being primed for 5 to 6 hours with $1 \mu \mathrm{g} / \mathrm{ml}$ LPS or Pam3CSK 4 in Opti-MEM (Life Technologies). Primed cells were transfected with $2 \mu \mathrm{g} / \mathrm{ml}$ LPS plus $0.25 \% \mathrm{v} / \mathrm{v}$ FuGENE HD (Promega).

MPO assay. Lungs were perfused with PBS to remove all blood, then weighed and frozen and stored at $-80^{\circ} \mathrm{C}$ for no more than 1 week before the MPO assay was performed. MPO activity was measured as previously described (4).

Immunoblotting. For immunoblotting, cells were lysed with RIPA buffer. Culture supernatants were precipitated with $7.2 \%$ trichloroacetic acid plus $0.15 \%$ sodium cholate. Protein concentrations were determined using Bradford (Bio-Rad). Proteins (20-50 $\mu \mathrm{g}$ ) were boiled, separated on SDS-PAGE, and transferred onto PVDF membranes (Bio-Rad). Membranes were blocked with 5\% nonfat dry milk in PBS with 0.1\% Tween 20 (PBST, Sigma-Aldrich) for 1 hour at room temperature and incubated overnight at $4^{\circ} \mathrm{C}$ with primary antibodies. Densitometry analysis was performed using Image J software (NIH), and the data were normalized against $\beta$-actin.

Measurement of pulmonary vessel filtration coefficient. Mice were anesthetized with an i.p. injection of ketamine $(100 \mathrm{mg} / \mathrm{kg}$ body weight) and xylazine (15 mg/kg body weight). Lungs were harvested and microvessel permeability was determined in isogravimetric lungs by determining microvascular filtration coefficient $\left(K_{\mathrm{f}_{\mathrm{c}}}\right)(50)$. In brief, outflow pressure was elevated by $10 \mathrm{~cm} \mathrm{H}_{2} \mathrm{O}$ for 20 minutes in isogravimetric perfused lungs. The lung wet weight gain during this time, which is the net fluid accumulation, was recorded. At the end of each experiment, lung dry weight was determined. $K_{f, c}$ (milliliters $\times \min ^{-1} \times$ centimeters $\mathrm{H}_{2} \mathrm{O} \times \mathrm{g}_{\text {dry }}$ weight ${ }^{-1}$ ) was calculated from the slope of the recorded weight change normalized to the pressure change and lung dry weight.

Evans blue-albumin pulmonary transvascular flux measurements. To measure vessel endothelial permeability, we performed Evans blue-albumin extravasation assay, which is a widely used and wellestablished in vivo method for a quantitative assessment of vascular permeability level (51). Physiological intact endothelium is permeable to water and ions and impermeable to proteins. Damaged endothelial barrier function by inflammatory stimuli results in protein leakage. By injecting Evans blue dye, which binds albumin, we can assess the extent of protein leakage from the blood stream. Briefly,
Evans blue-albumin was injected into anesthetized mice and allowed to circulate in the blood vessels for 30 minutes. Intravascular Evans blue was washed by PBS perfusion from the right ventricle for 2 minutes. Mouse lungs were excised, weighed, homogenized in $1 \mathrm{ml}$ PBS, and extracted overnight in $2 \mathrm{ml}$ formamide at $60^{\circ} \mathrm{C}$. Evans blue concentration in lung homogenate supernatants was quantified by the spectrophotometric method at absorbance of 620 and $740 \mathrm{~nm}$. Left lungs from the same mice used for Evans blue-albumin extravasation were excised and completely dried in the oven at $60^{\circ} \mathrm{C}$ overnight for calculation of lung wet/dry ratio.

Cytokine and chemokine measurements. Blood plasma and tissue homogenate from Casp $11^{+/+}$and Casp11 $11^{-/}$mice injected with LPS (40 $\mathrm{mg} / \mathrm{kg}$ ) or saline were analyzed for the presence of IL-1 $\beta$, IL-6, TNF- $\alpha$, and MIP-1 $\alpha$, using a commercially available ELISA kit (R\&D Systems).

$B M$ transplantation studies. Recipient mice were lethally irradiated with $10 \mathrm{~Gy}$ at a dose rate of $2 \mathrm{~Gy} / \mathrm{min}$. Before irradiation, the recipient mice were maintained on acidified, antibiotic water for 2 to 3 days. At 24 hours after irradiation, the mice were given a retroorbital intravenous injection of 3 million donor BM cells. BM cells were obtained by flushing tibia and femur cavities using cold RPMI 1640 containing 2\% FBS and $100 \mathrm{IU} / \mathrm{ml}$ penicillin and streptomycin. The cells were passed through a sterile $40-\mu \mathrm{m}$ nylon cell strainer, washed twice with serumfree RPMI 1640, and resuspended in HBSS (with $\mathrm{Ca}^{2+}$ and $\mathrm{Mg}^{2+}$ ) for injection. All subsequent experiments to assess endotoxemic responses were performed 6 to 8 weeks after BM transplantation.

LDH assay. hMVECs were cultured overnight in 96-well plates before being primed for 5 to 6 hours with $1 \mu \mathrm{g} / \mathrm{ml}$ LPS, Pam3CSK4, or $5 \mu \mathrm{g} / \mathrm{ml}$ poly(I:C) in Opti-MEM (Life Technologies). Primed cells were transfected with $2 \mu \mathrm{g} / \mathrm{ml}$ LPS using Lipofectamine 2000 reagent (Invitrogen) for 16 hours. ELISAs were used to measure IL-1 3 (R\&D Systems) in culture supernatants. A CytoTox 96 Non-Radioactive Cytotoxicity Assay (Promega) measured cell death according to the manufacturer's instructions.

Lung injury studies. For in vivo experiments, we used EC-specific caspase-11-deficient mice generated by tamoxifen ( $2 \mathrm{mg} / \mathrm{d}$ for 5 days) administered i.p. into Endo-SCL-Cre Casp11 ${ }^{f / f l}$ mice (C57BL/6 background), in which tamoxifen induced expression of a fusion protein of Cre recombinase with the modified estrogen receptor binding domain (Cre ERT2) under the control of the $\mathrm{Scl}$ promoter.

Mice received a single dose $(40 \mathrm{mg} / \mathrm{kg})$ of LPS (E. coli 0111:B4, InvivoGen) administered i.p. For histology, paraffin-embedded tissue sections were stained with H\&E or Leder stain.

Caspase-4/5 knockdown. hMVECs were nucleofected with $300 \mathrm{nM}$ siRNA according to the manufacturer's instructions (Amaxa Nucleofector Technology). The following validated siRNAs were used: CASP4, ON-TARGETplus SMART POOL Human CASP4 SiRNA (L-004404-00-0005, Dharmacon), CASP5, ON-TARGETplus SMART POOL Human CASP5 SiRNA (L-004405-02-0005, Dharmacon), and a nontargeting pool (Dharmacon).

MP analysis. Imaging cytometry was performed with the FlowSight Instrument (Amnis Corp.). Size calibration beads were purchased from Molecular Probes. All antibodies were purchased from BD Biosciences, unless otherwise stated, including anti-human CD31 AF647, anti-human CD41 FITC, anti-mouse CD31 APC, and anti-mouse CD41 FITC. Calibration beads were diluted in doublesterile-filtered $(0.22 \mu \mathrm{m})$ PBS after vortexing to disperse. Cytometry analysis was performed immediately after staining. 
For circulating plasma MP phenotyping experiments, $50 \mu$ l plateletpoor plasma (PPP) was stained with CD31-APC (endothelial marker) and CD41-FITC (platelet marker). Appropriate isotype and single stain controls were used. Samples were stained in the dark at room temperature for 30 minutes before acquisition with FlowSight. All samples were acquired on a FlowSight imaging cytometer, $\times 20$ magnification, with low flow rate/high sensitivity using INSPIRE software (MilliporeSigma). The percentage of MPs positive for each lineage marker was measured.

The instrument and INSPIRE software were set up as follows: channels 01 (bright field), 09 (bright field 2) and 06 (scattering channel) plus fluorescence channels required. Magnification was $\times 20$, and the lasers 488,642 , and 745 were activated for fluorescence and side scatter. The flow rate was set to low speed/high sensitivity, and stream alignment was adjusted where necessary. To obtain a collection gate, a scatter plot of bright field object area (channel 01) was plotted against side scatter (channel 06) intensity; this allow us to set a gate for size discrimination. Each gated population was interrogated via the image gallery to determine the upper and lower limits of MP size and shape. MPs characteristically appear as small and can be discriminated from cells, which are much larger, or cellular debris, which is not uniformly spherical.

To determine the concentration of the sample, IDEAS software (MilliporeSigma) was used. Raw image files with the entire number of events were opened. A scatter plot showing fluorescence intensity plotted against scattering intensity was generated and an MP gate reapplied and inspected visually to exclude inappropriate events. The gate was adjusted where necessary. The objects/ml feature was added to the analysis area and applied to the MP gate. To analyze the number of MPs generated, all events falling in the MP gate were plotted on a scatter graph showing time versus objects per second.

Patient samples. A total of 14 ARD patients (4 males, 3 females) and CPE patients ( 5 males, 2 females) from the intensive care unit of the Guangdong General Hospital who were receiving mechanical ventilation for ARD and CPE were enrolled. Data on demographic characteristics and medical history are provided in Supplemental Table 1. Blood gas analysis of all patients showed $\mathrm{PaO}_{2} / \mathrm{FiO}_{2}$ less than or equal to $300 \mathrm{mmHg}$ and APACHE II scores ranging from 14 to 32 . A total of 10 stable outpatients ( 7 males, 3 females) were also enrolled as a control group. We collected blood from a peripheral vein into heparin tubes that were centrifuged (Universal 16R) for 5 minutes at 3,000 $\mathrm{g}$ $\left(4^{\circ} \mathrm{C}\right)$. The plasma supernatant was removed from the spun samples and frozen at $-80^{\circ} \mathrm{C}$ until the time of MP analysis.

Statistics. Data were analyzed by 2-tailed unpaired Student's $t$ test for comparisons of 2 groups or 1-way ANOVA of the repeated experiments followed by the Tukey's post hoc pairwise multiple comparisons when appropriate with Prism 7 (GraphPad). $P<0.05$ was considered significant. For all bar graphs, the mean \pm SEM is plotted. All in vitro experiments were repeated at least 3 times unless otherwise indicated.

Study approval. The Animal Care Committee and Institutional Biosafety Committee of the University of Illinois at Chicago approved all mouse protocols. The human study was approved by the Medical Ethics Committee of Guangdong General Hospital. Written informed consent was required from participants or their surrogates. Patients with surrogate consents were reconsented if they regained decisionmaking capacity during the hospitalization.

\section{Author contributions}

ABM conceived the study. KTC, SX, JR, and ABM designed the experiments. KTC, SX, ZY, ZH, KMT, XG, SMV, SA, AD, and MM performed the experiments and analyzed the data. KTC, SX, and JR wrote the initial draft of the paper. EAM and ABM critically revised the manuscript. All authors reviewed and edited the manuscript.

\section{Acknowledgments}

The work was supported by NIH grants R01-HL45638 (to ABM), P01-HL60678 (to ABM), P01-HL077806 (to ABM), T32-HL007829 (to ABM), R01-HL118068 (to JR and ABM), R01-HL90152 (to JR and ABM), R01-AI097518 (to EAM), and R01-AI119073 (to EAM) and the Yang Biomedical Scholars Award (to EAM).

Address correspondence to: Asrar B. Malik or Jalees Rehman, University of Illinois College of Medicine, Department of Pharmacology, 835 S. Wolcott Avenue, Mailcode 868, Chicago, Illinois 60612, USA. Phone: 312.996.7636; Email: abmalik@uic.edu (A.B. Malik); jalees@uic.edu (J. Rehman).
1. Ware LB, Matthay MA. The acute respiratory distress syndrome. $N$ Engl J Med. 2000;342(18):1334-1349.

2. Matthay MA, Ware LB, Zimmerman GA. The acute respiratory distress syndrome. J Clin Invest. 2012;122(8):2731-2740.

3. Mehta D, Malik AB. Signaling mechanisms regulating endothelial permeability. Physiol Rev. 2006;86(1):279-367.

4. Gong H, et al. HIF $2 \alpha$ signaling inhibits adherens junctional disruption in acute lung injury. J Clin Invest. 2015;125(2):652-664.

5. Maniatis NA, Orfanos SE. The endothelium in acute lung injury/acute respiratory distress syndrome. Curr Opin Crit Care. 2008;14(1):22-30.

6. Nourshargh S, Alon R. Leukocyte migration into inflamed tissues. Immunity. 2014;41(5):694-707.

7. Baumgartner JD, et al. Prevention of gramnegative shock and death in surgical patients by antibody to endotoxin core glycolipid. Lancet.
1985;2(8446):59-63.

8. Medzhitov R, Preston-Hurlburt P, Janeway CA. A human homologue of the Drosophila Toll protein signals activation of adaptive immunity. Nature. 1997;388(6640):394-397.

9. Poltorak A, et al. Defective LPS signaling in $\mathrm{C} 3 \mathrm{H} /$ $\mathrm{HeJ}$ and C57BL/10ScCr mice: mutations in Tlr4 gene. Science. 1998;282(5396):2085-2088.

10. Hagar JA, Powell DA, Aachoui Y, Ernst RK, Miao EA. Cytoplasmic LPS activates caspase-11: implications in TLR4-independent endotoxic shock. Science. 2013;341(6151):1250-1253.

11. Kayagaki N, et al. Noncanonical inflammasome activation by intracellular LPS independent of TLR4. Science. 2013;341(6151):1246-1249.

12. Kayagaki N, et al. Caspase-11 cleaves gasdermin $\mathrm{D}$ for non-canonical inflammasome signalling. Nature. 2015;526(7575):666-671.

13. Jorgensen I, Miao EA. Pyroptotic cell death defends against intracellular pathogens. Immunol
Rev. 2015;265(1):130-142.

14. Shi J, et al. Cleavage of GSDMD by inflammatory caspases determines pyroptotic cell death. Nature. 2015;526(7575):660-665.

15. Ding J, et al. Pore-forming activity and structural autoinhibition of the gasdermin family. Nature. 2016;535(7610):111-116.

16. Sborgi L, et al. GSDMD membrane pore formation constitutes the mechanism of pyroptotic cell death. EMBO J. 2016;35(16):1766-1778.

17. Liu $X$, et al. Inflammasome-activated gasdermin D causes pyroptosis by forming membrane pores. Nature. 2016;535(7610):153-158.

18. Aglietti RA, et al. GsdmD p30 elicited by caspase-11 during pyroptosis forms pores in membranes. Proc Natl Acad Sci U S A. 2016;113(28):7858-7863.

19. Broz P. Immunology: Caspase target drives pyroptosis. Nature. 2015;526(7575):642-643

20. de Gassart A, Martinon F. Pyroptosis: caspase-11 
unlocks the gates of death. Immunity. 2015;43(5):835-837.

21. Yuan J, Najafov A, Py BF. Roles of caspases in necrotic cell death. Cell. 2016;167(7):1693-1704.

22. Shi J, et al. Inflammatory caspases are innate immune receptors for intracellular LPS. Nature. 2014;514(7521):187-192.

23. Vanaja SK, et al. Bacterial outer membrane vesicles mediate cytosolic localization of LPS and caspase-11 activation. Cell. 2016;165(5):1106-1119.

24. Man SM, et al. IRGB10 liberates bacterial ligands for sensing by the AIM2 and caspase-11NLRP3 inflammasomes. Cell. 2016;167 (2):382-396.e17.

25. Wallach D, Kang TB, Dillon CP, Green DR. Programmed necrosis in inflammation: Toward identification of the effector molecules. Science. 2016;352(6281):aaf2154.

26. Fink SL, Cookson BT. Apoptosis, pyroptosis, and necrosis: mechanistic description of dead and dying eukaryotic cells. Infect Immun. 2005;73(4):1907-1916.

27. Grootjans S, Vanden Berghe T, Vandenabeele P. Initiation and execution mechanisms of necroptosis: an overview. Cell Death Differ. 2017;24(7):1184-1195.

28. Aachoui $Y$, et al. Caspase-11 protects against bacteria that escape the vacuole. Science. 2013;339(6122):975-978.

29. Maltez VI, et al. Inflammasomes coordinate pyroptosis and natural killer cell cytotoxicity to clear infection by a ubiquitous environmental bacterium. Immunity. 2015;43(5):987-997.

30. Jorgensen I, Zhang Y, Krantz BA, Miao EA. Pyroptosis triggers pore-induced intracellular traps (PITs) that capture bacteria and lead to their clearance by efferocytosis. JExp Med. 2016;213(10):2113-2128.
31. Kayagaki N, et al. Non-canonical inflammasome activation targets caspase-11. Nature. 2011;479(7371):117-121.

32. Miao EA, Rajan JV, Aderem A. Caspase-1induced pyroptotic cell death. Immunol Rev. 2011;243(1):206-214.

33. Broz P, Dixit VM. Inflammasomes: mechanism of assembly, regulation and signalling. Nat Rev Immunol. 2016;16(7):407-420.

34. Man SM, Kanneganti TD. Converging roles of caspases in inflammasome activation, cell death and innate immunity. Nat Rev Immunol. 2016;16(1):7-21.

35. Napier BA, et al. Complement pathway amplifies caspase-11-dependent cell death and endotoxin-induced sepsis severity. J Exp Med. 2016;213(11):2365-2382.

36. Zanoni I, et al. An endogenous caspase-11 ligand elicits interleukin-1 release from living dendritic cells. Science. 2016;352(6290):1232-1236.

37. Ralston KS, et al. Trogocytosis by Entamoeba histolytica contributes to cell killing and tissue invasion. Nature. 2014;508(7497):526-530.

38. Aachoui $Y$, et al. Canonical inflammasomes drive IFN- $\gamma$ to prime caspase-11 in defense against a cytosol-invasive bacterium. Cell Host Microbe. 2015;18(3):320-332.

39. Lin XY, Choi MS, Porter AG. Expression analysis of the human caspase- 1 subfamily reveals specific regulation of the CASP5 gene by lipopolysaccharide and interferon-gamma. J Biol Chem. 2000;275(51):39920-39926.

40. Göthert JR, et al. Genetically tagging endothelial cells in vivo: bone marrow-derived cells do not contribute to tumor endothelium. Blood. 2004;104(6):1769-1777.

41. Bergsbaken T, Fink SL, Cookson BT. Pyroptosis: host cell death and inflammation. Nat Rev Micro- biol. 2009;7(2):99-109.

42. Aird WC. The role of the endothelium in severe sepsis and multiple organ dysfunction syndrome. Blood. 2003;101(10):3765-3777.

43. Andonegui G, et al. Endothelium-derived Tolllike receptor- 4 is the key molecule in LPSinduced neutrophil sequestration into lungs. J Clin Invest. 2003;111(7):1011-1020.

44. Iwasaki A, Medzhitov R. Control of adaptive immunity by the innate immune system. $\mathrm{Nat}$ Immunol. 2015;16(4):343-353.

45. Zafrani L, et al. Calpastatin controls polymicrobial sepsis by limiting procoagulant microparticle release. Am J Respir Crit Care Med. 2012;185(7):744-755.

46. Combes V, et al. In vitro generation of endothelial microparticles and possible prothrombotic activity in patients with lupus anticoagulant. J Clin Invest. 1999;104(1):93-102.

47. Mause SF, Weber C. Microparticles: protagonists of a novel communication network for intercellular information exchange. Circ Res. 2010;107(9):1047-1057.

48. Ghosh A, et al. Platelet CD36 mediates interactions with endothelial cell-derived microparticles and contributes to thrombosis in mice. JClin Invest. 2008;118(5):1934-1943.

49. Headland SE, Jones HR, D'Sa AS, Perretti M, Norling LV. Cutting-edge analysis of extracellular microparticles using ImageStream $(\mathrm{X})$ imaging flow cytometry. Sci Rep. 2014;4:5237.

50. Vogel SM, et al. Abrogation of thrombin-induced increase in pulmonary microvascular permeability in PAR-1 knockout mice. Physiol Genomics. 2000;4(2):137-145.

51. Radu M, Chernoff J. An in vivo assay to test blood vessel permeability. J Vis Exp. 2013;16;(73):e50062. 